healthcare elsewhere. This is of more concern for those at higher STI risk for example the younger, MSM and those with concurrent partners, although higher proportions of younger people reported Chlamydia testing elsewhere. We used unsafe sex as one measure of exposure to risk, which does not take into account characteristics of partner(s) and the wider sexual network. Further improvements are necessary to reach those at-risk, including through effective, diverse service provision.

\section{P4.61 SEXUAL IDENTITY, ATTRACTION AND EXPERIENCE IN BRITAIN: THE IMPLICATIONS OF USING DIFFERENT DIMENSIONS OF SEXUAL ORIENTATION TO ESTIMATE THE SIZE OF SEXUAL MINORITY POPULATIONS}

${ }^{1}$ Mercer Ch, ${ }^{2} \mathrm{R}$ Geary, ${ }^{1} \mathrm{C}$ Tanton, ${ }^{2} \mathrm{~B}$ Erens, ${ }^{1} \mathrm{~S}$ Clifton, ${ }^{3}$ Mitchell $\mathrm{Kr},{ }^{1} \mathrm{P}$ Sonnenberg. ${ }^{1} \mathrm{UCL}$, London, UK; ${ }^{2}$ LSHTM, London, UK; ${ }^{3}$ University of Glasgow, Glasgow, UK

\subsection{6/sextrans-2017-053264.557}

Introduction Sexual orientation encompasses three dimensions: sexual identity, attraction and behaviour. Many health and policy surveys and inequality monitoring activities collect data only on identity, while STI risk is primarily driven by behaviour. We present estimates of all three dimensions and examine the extent of their overlap, for men and women, and consider the implications of using the different criteria to estimate the size of sexual minority populations in Britain.

Methods Descriptive analyses of data from Britain's third National Survey of Sexual Attitudes and Lifestyles, a probability survey (15 162 people aged 16-74 years) undertaken 2010-2012 using a computer-assisted personal- and self-interviewing (CAPI/CASI). A standard question was used to define sexual identity (CAPI). Participants were also asked to describe their sexual attraction according to a scale ranging from exclusively opposite-sex to exclusively same-sex (CAPI). Same-sex sex was defined as ever having had sex (including genital contact) with someone of the same sex (CASI).

Results A lesbian, gay or bisexual (LGB) identity was reported by $2.5 \%$ of men and $2.4 \%$ of women, whilst $6.5 \%$ of men and $11.5 \%$ of women reported any same-sex attraction, and $5.5 \%$ of men and $6.1 \%$ of women reported ever experience of same-sex sex. This equates to approximately 573000 men and 559000 women in Britain currently self-identifying as LGB, less than half the number who are estimated to have ever had same-sex sex: 1,262,000 men and 1,422,000 women. Of those reporting having same-sex sex in the past 5 years, $28 \%$ of men and $45 \%$ of women identified as heterosexual.

Conclusions Substantial incongruity exists between the three measures of sexual orientation on an individual level, particularly for women. The size of sexual minority populations will depend on the dimension of sexual orientation applied, the choice of which depends on context and purpose. Regardless, the decision to use a particular dimension should be made explicitly, with a clear rationale, and with awareness of the limitations of each.

\section{P4.62 HIV TESTING PREFERENCES AMONG LONG DISTANCE TRUCK DRIVERS IN KENYA: A DISCRETE CHOICE EXPERIMENT}

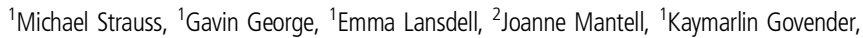
${ }^{3}$ Matthew Romo, ${ }^{4}$ Jacob Odhiambo, ${ }^{4}$ Eva Mwai, ${ }^{4}$ Eston Nyaga, ${ }^{3}$ Elizabeth Kelvin. ${ }^{1}$ Health Economics and HIV and AIDS Research Division, University of Kwazulu-Natal, Durban, South African Republic; ${ }^{2}$ HIV Centre for Clinical and Behavioural Studies, Department of Psychiatry, Division of Gender, New York, USA; ${ }^{3}$ Department of Epidemiology and Biostatistics, Cuny Graduate School of Public Health and Health Poli, New York, USA; ${ }^{4}$ North Star Alliance, Nairobi, Kenya

\subsection{6/sextrans-2017-053264.558}

Introduction Providing HIV testing to truck drivers in Africa is crucial but challenging. The introduction of HIV self-testing provides expanded service delivery options for clients, potentially increasing demand for services and expanding coverage - especially important for high-risk and difficult-to-reach populations. This study examines the preferences regarding HIV testing service delivery models, among long distance truck drivers in an effort to identify testing services that would appeal to this population.

Methods Using a discrete choice experiment, this study examines the drivers of choice regarding HIV counselling and testing among 305 truck drivers recruited from two roadside wellness clinics along major trucking routes in Kenya. Participants made trade-offs between characteristics of HIV testing service delivery models by making hypothetical choices in a series of paired HIV testing scenarios. Conditional logit models were used to identify the HIV testing characteristics driving the selection of preferred scenarios, and determine whether preferences interact with individual characteristics especially HIV testing history.

Results Participants preferred free, provider-administered HIV testing at a roadside clinic, using a finger-prick test, with inperson counselling, undertaken in the shortest possible time. The strongest driver of choice was the cost of the test. Those who had never tested previously preferred oral testing and telephonic counselling, while those who were not regular testers favoured clinic based- over self-testing.

Conclusion The results of this study indicate that for the majority of participants - most of whom had tested before the existing services offered at roadside clinics were the preferred service delivery model. The introduction of oral selftesting increases the options available to truck drivers and may even improve testing uptake for some, especially among those who have never tested before. However, these findings suggest the impact on HIV testing uptake of introducing oral self-testing may be limited in this population.

\section{P4.63 DESIGNING HUMAN IMMUNODEFICIENCY VIRUS COUNSELLING AND TESTING SERVICES TO MAXIMISE UPTAKE AMONG HIGH SCHOOL LEARNERS IN SOUTH AFRICA: WHAT MATTERS?}

${ }^{1}$ Michael Strauss, ${ }^{1}$ Gavin George, ${ }^{2}$ Bruce Rhodes. ${ }^{1}$ Health Economics and HIV and AIDS Research Division, University of Kwazulu-Natal, Durban, South African Republic; ${ }^{2}$ School of Accounting, Economics and Finance, University of Kwazulu-Natal, Durban, South African Republic

10.1136/sextrans-2017-053264.559 
Introduction Increasing human immunodeficiency virus (HIV) testing in South Africa is vital for the HIV response. Targeting young people is important as they become sexually active and because HIV risk rapidly increases as youth enter their 20s. This study aims to increase the understanding of high school learners' preferences regarding the characteristics of HIV testing service delivery models and to inform policy makers and implementers regarding potential barriers to and facilitators of HIV testing.

Methods An attitudinal survey was used to examine HIV testing prefer- ences among 248 high school learners in KwaZulu-Natal. Statistical tests were used to identify the most favoured characteristics of testing service delivery models and examine key differences in preferences based on demographic characteristics and testing history.

Results Most learners were found to prefer testing offered at a clinic on a Saturday (43\%), using a finger prick test (59\%), conducted by a doctor (61\%) who also provides individual counselling $(60 \%)$. Shorter testing times were preferred, as well as a monetary incentive to cover any associ- ated expenses. Time, location, the type of test, and who conducts the test were most important. However, stratified analysis suggests that preferences diverge, particularly around gender, grade, but also sexual history and previous testing experience. Conclusion Human immunodeficiency virus testing services can be improved in line with preferences, but there is no single optimal design that caters to the preferences of all learners. It is unlikely that a "one-size-fits-all" approach will be effective to reach HIV testing targets. A range of options may be required to maximise coverage.

\section{P4.64 ATTITUDINAL AND BEHAVIOURAL DIFFERENCES BETWEEN YOUTH WHO HAVE HAD ANAL SEX AND THOSE WHO HAVE NOT IN CAPE TOWN, SOUTH AFRICA}

${ }^{1}$ Michele Ybarra, ${ }^{1}$ Myeshia Price-Feeney, ${ }^{2}$ Kelvin Mwaba. ${ }^{1}$ Centre for Innovative Public Health Research, San Clemente, USA; ${ }^{2}$ Univeristy of Western Cape, Cape Town, South African Republic

\subsection{6/sextrans-2017-053264.560}

Introduction Although HIV rates among South African youth are among the highest in the world, most research efforts have overlooked anal sex as a contextualising factor.

Methods In 2012, a pencil-and-paper survey was completed by 937 youth 16 years of age and older who were attending low-income secondary schools in Cape Town.

Results Eleven and $31 \%$ of female and male youth, respectively, reported ever having anal sex. Among sexually active male and female respondents, those who had ever had anal sex were more likely to report inconsistent condom use, perpetrate dating violence, and experience victimisation and perpetration of sexually coercive behaviour. Female respondents who had ever had anal sex had significantly lower levels of HIV information than sexually active females who had not had anal sex.

Conclusion Rates of anal sex are higher for boys than girls in the survey, yet the associated risk factors were strikingly similar. Harm reduction strategies for anal sex should be made available to South African youth.

\section{P4.65 LESSONS LEARNED USING FB TO RECRUIT LGBT ADULTS ACROSS EASTERN AFRICA INTO ONLINE SEXUAL HEALTH FOCUS GROUPS}

Michele Ybarra, Emilie Chen, Tonya Prescott. Centre for Innovative Public Health Research, San Clemente, USA

\subsection{6/sextrans-2017-053264.561}

Introduction Because of cultural and legal discrimination enacted towards LGBT people in eastern Africa, innovative methods are needed to safely engage LGBT Africans in sexual health research.

Methods We conducted two online focus groups (FGs) with eastern African MSM, one in December 2016 and the other in January 2017. Participants were recruited via Facebook advertisements, which were targeted to men who: were 'interested in' men or were 'interested in' men and women; were living in: Rwanda, Uganda, Kenya, Tanzania, or South Sudan; and were 18 years and older. People who clicked on the ad were linked to the study website, which explained the FG activity, provided an eligibility screener, and a consent form for those who were eligible. Once consented, MSM were linked to the online FG, where they could anonymously post answers.

Results After a 27 hour FB campaign, 176480 people were reached across eastern Africa, 11889 of whom clicked on the ad. Most clicks (59\%) were from people in Tanzania; Kenya (22\%) and Uganda (13\%) were the next most common sources of clicks. Thirty-three people completed a screener and were eligible, and 22 consented to take part in the first FG. Of those who consented, 5 people posted in at least one thread in the online FG bulletin board. Of the 15 threads we posted, 3 received posts from participants, none of which were sexual health-related. To invigorate response rates, the FG script was shortened from 15 to 6 threads and displayed on one instead of three separate pages. A second 27 hour FB campaign was launched. A similar number of eligible people were identified $(n=35)$ while more consented $(n=34)$. One person went on to post in 4 sexual health-related threads, and another posted in one.Additional FGs are being conducted. Lessons learned that can inform future research that endeavours to engage LGBT Africans will be presented.

Conclusion Although online data collection holds promise in reaching hidden populations, piloting work is necessary to determine the most effective way to ensure privacy and promote participation.

\section{P4.66 OPPORTUNITIES TO TAILOR HIV PREVENTION PROGRAMMING FOR UGANDAN YOUNG ADULTS}

${ }^{1}$ Michele Ybarra, ${ }^{1}$ Tonya Prescott, ${ }^{2}$ Emmanuel Kyagaba. ${ }^{~}$ Centre for Innovative Public Health Research, San Clemente, USA; ${ }^{2}$ Internet Solutions for Kids, Uganda; Mbarara University of Science and Technology, Mbarara, Uganda

\subsection{6/sextrans-2017-053264.562}

Introduction Uganda, like most Sub-Saharan African countries, has comprehensive HIV prevention campaigns, yet programs tailored to young adults are completely lacking.

Methods In 2016, 202 18-22 year olds were recruited from across Uganda via Facebook to take part in online, 3 day, asynchronous focus groups (FGs). The aim of the FGs was to understand sexual health decision making to inform HIV prevention content development. The study sample was 
purposefully balanced on age, district, educational attainment, employment status, and income. Eight FGs were conducted; participants were stratified by sex and sexual behaviour in the past 12 months.

Results Yong adults who were abstinent said they were making this choice because they treasured virginity; and wanted to stay STD-free and avoid pregnancies. There were drawbacks, particularly for women who said that their boyfriends may leave if they did not have sex. Peer pressure and feeling excluded from those who were having sex were also disadvantages.

Ugandan young adults talked about the cultural norm of having multiple partners, both for financial gain and social status. Using condoms was universally noted as the right thing to do. At the same time, sexually active youth voiced several barriers to condom use, including: being allergic to rubber, fear of getting cancer with frequent use, and embarrassment. When asked about how confident they were in negotiating condoms, sexually experienced women voiced a range or responses from 'I can't at all' to 'sometimes' to 'always'.Refusing sex was possible: If a woman did not want to have sex, respondents said she could blame it on her period and if a man did not want to have sex, he could say he was 'tired'. That said, all agreed that the refusal of sex in a relationship could be met with consequences (e.g., distrust by one's partner, an end to the relationship).

Conclusion Several important challenges that Uganda young adults faces in making healthy sexual decisions were voiced. These issues need to be directly addressed in HIV prevention programs tailored to this age group.

\section{P4.67 STI TESTING BEHAVIOUR AMONG SEXUAL MINORITY ADOLESCENT WOMEN RECRUITED FROM FB IN THE UNITED STATES}

${ }^{1}$ Michele Ybarra, ${ }^{1}$ Tonya Prescott, ${ }^{1}$ Myeshia Price-Feeney, ${ }^{2}$ Elizabeth Saewyc, ${ }^{3}$ Margaret Rosario. ${ }^{1}$ Centre for Innovative Public Health Research, San Clemente, USA; ${ }^{2}$ University of British Columbia, Vancouver, USA; ${ }^{3}$ City University of New York, New York City, USA

\subsection{6/sextrans-2017-053264.563}

Introduction Despite what adolescent health practitioners may assume, multiple studies have found that lesbian, gay, and bisexual (LGB) girls are more likely than heterosexual girls to have unprotected penile-vaginal sex, engage in penile-anal sex, have multiple sexual partners, have high risk sexual partners, and to get pregnant. In the face of these disparities, it is unknown if LGB girls are getting tested for STIs.

Methods Ninety-one 14-18 year old cisgender females who identified as sexual minority (i.e., had a sexual identity other than exclusively heterosexual) and lived in the United States were recruited through Facebook and surveyed online in July 2016.

Results Twenty-nine percent of respondents estimated they lived in a lower than average income household. Seventy-five percent were White race; $11 \%$ were Hispanic ethnicity. Two in five respondents (42\%) lived in a suburban area, one in four (27\%) lived in an urban area, and just under one in three $(30 \%)$ lived in a small town or rural area.LGB girls reported rates of lifetime STI testing (21\%) similar to rates of HIV testing (19\%). Four percent said they had ever tested positive for an STI; none reported a positive HIV test.Compared to $6 \%$ of LGB girls who have only had sex with girls,
$30 \%$ of LGB girls who have only had sex with guys, and $46 \%$ of girls who have had sex with guys and girls have ever been tested for an STI $(p=0.01)$.Although not statistically significant, LGB girls who did not use a condom at last penilevaginal sex $(62 \%)$ were more likely than those who did $(35 \%)$ to have ever been tested for STIs $(p=0.15)$.

Conclusion It may be that LGB girls who have male partners, either exclusively or while also having female partners, are aware of the STI risk that penile-vaginal sex may confer and are testing accordingly. The higher rates of STI testing in the face of lower condom use however, may suggest that some LGB teens who are having sex with guys are using testing instead of condoms as a form of STI prevention.

\section{P4.68 INTERNALISED OF HIV-RELATED STIGMA AND ASSOCIATED FACTORS AMONG HIV-INFECTED ADULTS RECEIVING CARE}

${ }^{1}$ Mina Qobadi, ${ }^{1}$ Ali Dehghanifirouzabadi, ${ }^{2}$ Tiffany Mcdonald, ${ }^{1}$ Tametria Samms, ${ }^{1}$ Kendra Johnson. ${ }^{1}$ Mississippi State Department of Health, Jackson, USA; ${ }^{2}$ Mississippi State Department of Health, Jacskon, USA

\subsection{6/sextrans-2017-053264.564}

Introduction Internalisation of HIV-related stigma may affect a person's disease management and his her accessibility to services. However, little is known about HIV-related stigma and its associated factors in patients with HIV. We aimed to examine HIV-related stigma and its associated factors.

Methods We used Mississippi Medical Monitoring Project (MMP) data from 2011 to $2014(\mathrm{n}=793)$. MMP is a crosssectional surveillance system designed to assess the behavioural and clinical characteristics of HIV-infected adults at least 18 years of age receiving outpatient care in the United States. Dependent variable was HIV-related stigma. Our independent variables were gender, sexual orientation, race, age, education, poverty level, smoking, binge drinking, drug abuse, length of time diagnosed with HIV, and insurance. T-test, one-way ANOVA, and multiple linear regression were conducted $(\mathrm{p}<0.05)$.

Results Overall, 75\% of respondents reported at least one internalised HIV-related stigma experience. The average stigma score overall was 2.3 (out of 6), with a standard error of 0.07. Our finding showed that stigma was significantly higher among females $(\mathrm{M}=2.6, \mathrm{t}=2.9, \mathrm{p}=0.003)$, patients aged 18$24(\mathrm{M}=2.9, \mathrm{~F}=4.6, \mathrm{p}=0.003)$, those with less than high school education $(\mathrm{M}=2.7, \mathrm{t}=2.4, \mathrm{p}=0.02)$, binge drinker $(\mathrm{M}=2.8, \quad \mathrm{t}=2.3, \quad \mathrm{p}=0.02)$, drug abuser $(\mathrm{M}=2.7, \mathrm{t}=2.3$, $\mathrm{p}=0.02$ ), those who have been diagnosed with HIV since $<5$ years ago $(\mathrm{M}=2.6, \mathrm{~F}=6.6, \mathrm{p}=0.001)$, and those who had private insurance $(\mathrm{M}=2.8, \mathrm{~F}=5.5, \mathrm{p}=0.004)$. Multiple linear regression analysis showed that being female $(\beta=0.52)$, having less than high school education $(\beta=0.55)$, drug abusing $(\beta=0.66)$, and having private insurance $(\beta=0.77)$ contribute positively to predict experiencing stigma after entering all variables together.

Conclusion The findings indicate that HIV-related stigma is very common among HIV patients, and is significantly associated with gender, education, drug abuse and insurance status, highlighting a need for stigma reduction interventions, with a focus on population at risk. 


\section{P4.69 HEALTH CARE SEEKING BEHAVIOUR FOR SYMPTOMS OF STIS/HIV AMONG THE TRANSGENDER IN MUMBAI, INDIA}

Mithlesh Chourase. International Institute for Population Sciences (IIPS), Mumbai, Mumbai, India

10.1136/sextrans-2017-053264.565

Introduction Health of the transgender is as important as any other population sub-groups. However, little is known about the issues of health problems and health seeking behaviour of transgender in India. One of the major risk factors for acquisition of HIV and other STIs for MTF transgender persons is Commercial sex workers (CSW). As with HIV infection in this population, it is difficult to ascertain the exact prevalence of CSW in MTF transgender persons. This study is an attempt to assess the health care seeking behaviour of women reporting STIs/HIV symptoms in mumbai, India and to examine the association of background sociodemographic characteristics with Health care seeking behaviour.

Methodology The study used the primary survey data conducted in Mumbai city among the transgender community with a total sample of 120 . Both qualitative and quantitative data was collected on demographic and socio-economic characteristic, sexual health problems and health seeking behaviour among transgender.

Results Among the transgender, the prevalence of sexual health is very high. In this community $28 \%$ and $21.7 \%$ of the transgender are suffered from STI and HIV respectively. Among those who suffered from any sexual health problems, $7 \%$ of the transgender do not take any treatment. Those who seek treatment for sexual health problems most of them go to government medical institution because of low treatment cost compared to a private institution. On the other hand, those who don't seek treatment revealed that they don't prefer to take treatment because of their personal problem or due to shame, high medicine cost and few believe in yoga.

Conclusion Though the majority of transgender heard about STI, the correct knowledge of sexual health transmission is lacking among the transgender. There is a need for increasing awareness regarding STI/HIV and their sequelae. Targeted health education programmes should be necessary to improve health care seeking among transgender.

\section{P4.70 AVERTING AN IMPENDING HIV EPIDEMIC: MASSIVE INTERVENTIONS NEEDED FOR INJECTING DRUG USERS (IDUS) IN BANGLADESH}

Mohammed Abul Kalam. Siam Health Care, Dhaka, Bangladesh

10.1136/sextrans-2017-053264.566

Introduction Far from being an isolated population, injectors are intimately connected with others. They are linked with the rest of society - they have regular sex partners, they buy sex from women, as well as other men, they sell blood, and they also move between cities and inject. As long as HIV prevention through safe behaviour is not norm, the rest society is at risk from this virus. This study aims to collect information on IDUs and their health seeking behaviour in Bangladesh.

Methods A cross-sectional study was conducted among 120 IDUs attending a drug rehabilitation centre in Rajshahi, Bangladesh. Data were collected on socio-demographics, drug use, health seeking behaviour, knowledge of injecting drugs, and sexual behaviour.

Results The mean \pm SD and median (IQR) age of the participants were $32.5 \pm 21.3$ and 33 (27-38) years, respectively, with only $9.2 \%$ females. Injection buprenorphine was the drug of choice for $40 \%$ of participants, and $58 \%$ of the participants first started drug use with smoking cannabis. $73.3 \%$ of participants shared needles sometimes and $57.5 \%$ were willing to use the needle exchange programs. $60 \%$ of the participants had no knowledge about the diseases spread by injection. Condom use during the last intercourse with regular partners was $11.7 \%$ and with any partners $15.8 \%$.

Conclusion IDUs in Bangladesh are a high-risk group for HIV/ AIDS due to lack of knowledge and risky behaviour. Bangladesh must immediately make it high priority to scale up interventions massively and stop further spread of HIV among drug injectors and other vulnerable people. And Bangladesh must act rapidly and decisively to avert the impending HIV epidemic or it will be too late.

\section{P4.71 THE POWER OF PEOPLE LIVING WITH HIV (PLHIV) IN STIGMA REDUCTION}

Mohammed Ahmed Taha Elzaki. UNFPA, Khartoum, Sudan

10.1136/sextrans-2017-053264.567

This paper briefly reflects the fact that People living with HIV/ AIDS (PLHIV) can be potential resources for HIV-related stigma reduction, the paper shares the experience of Sudanese People living With HIV/AIDS Care Association (SPCA) on reducing selfsigma among the PLHIV themselves and on the other hand reducing the stigma among health service providers and the general population. In the near past, Stigma was the major challenge constraining HIV intervention efforts in Sudan where the social context is one of the generating factor for the stigma and discrimination, the type of stigma in Sudan was the most furious one which influence PLHIV and their families, it was a complex model of stigma that deny PLHIV to even access care and treatment. The efforts made to empower PLHIV were a breakthrough towards better PLHIV-friendly environment free of stigma and discrimination. This paper focuses on the impact of the PLHIV efforts in the stigma reduction, which has contributed to put PLHIV and AIDS issues in the primary agenda of the government commitment and helped to advocate for supporting the overall intervention of AIDS in the country. The paper also shows how far the role of other sectors contributed to empower PLHIV and helped the national AIDS Programme to apply mature strategies on stigma reduction and comprehensive programming for HIV response.

\section{P4.72 STIGMA AND DISCRIMINATION EXPERIENCED BY YOUNG MEN WHO HAVE SEX WITH MEN IN LARGE CITIES OF MYANMAR}

Myo Myo Mon, Kyaw Min Htut, Wai Wai Myint. Ministry of Health and Sports, Yangon, Myanmar Burma

\subsection{6/sextrans-2017-053264.568}

Introduction Young men who have sex with men (YMSM) are experiencing stigma and discrimination ( $S$ and $D)$ resulting in social exclusion with adverse consequences. Therefore, a study 